\title{
EL ANÁLISIS DE SEMEJANZA DE LAS PROPIEDADES
}

\author{
Joan PAGÈS \\ Departament de Filologia i Filosofia \\ Facultat de Lletres. Universitat de Girona
}

Una teoría de las propiedades debe dar una explicación de tres tipos de hechos distintos, los cuales, sin embargo, mantienen estrechas conexiones entre sí. En primer lugar, debe dar cuenta de los hechos de ejemplificación, consistentes en que cierto particular tiene (o ejemplifica) cierta propiedad. Así, son hechos de ejemplificación que cierto diamante tenga un índice de refracción de 2,419, o que la sal sea soluble en azúcar. En segundo lugar, tenemos la identidad de tipos, que concierne a hechos en que dos particulares tienen una propiedad en común. Son casos de identidad de tipos el hecho de que ciertas dos esmeraldas sean verdes, o que una muestra de sal y una de azúcar sean solubles. Finalmente, tenemos hechos de semejanza, que involucran a dos particulares que se asemejan (en cierto aspecto). De este modo, decimos que dos esmeraldas se asemejan (en el aspecto "color»), o que una muestra de sal y otra de azúcar se asemejan (en el aspecto «solubilidad»).

La mayoría de las teorías de las propiedades rivales del análisis de semejanza explotan los vínculos existentes entre estos tres tipos de hecho del mismo modo. Se trata de un aspecto común a teorías que, por otra parte, involucran compromisos metafísicos ciertamente dispares. Tanto las teorías nominalistas que apelan a meros particulares, ya sean predicados, conceptos, agregados o clases, como las teorías de corte realista que emplean tropos o universales, hallamos la misma estrategia explicativa. En primer lugar, todas estas teorías alternativas seleccionan los hechos de ejemplificación como aquella clase de hechos que recibirá una explicación básica, distinta en cada teoría. Por ejemplo, el nominalismo de clases da cuenta de los hechos de ejemplificación del tipo indicado en los términos siguientes: $a$ ejemplifica un propiedad P si y sólo si a pertenece a la clase de los 
Ps. En segundo lugar, se procede a explicar los otros dos tipos de hechos en términos del primero. El expediente es el siguiente: los hechos de semejanza se explican en términos de la identidad de tipos, mientras que la identidad de tipos se explica, a su vez y de modo inmediato, en términos de hechos de ejemplificación. Así, el hecho de que dos esmeraldas, $a$ y $b$, se asemejen (en el aspecto "color") se explica en términos del hecho de que ambas esmeraldas, $a$ y $b$, tienen en común la propiedad de ser verdes, el cual se explica a su vez mediante el hecho de que la esmeralda a tiene la propiedad de ser verde y el hecho de que la esmeralda $b$ tiene la propiedad de ser verde.

Por su parte, el análisis de semejanza, aunque también explota los vínculos existentes entre estos tres tipos de hechos, opera en la dirección inversa desde el punto de vista explicativo, y prescinde de dar una explicación del hecho que toma como el más básico de los tres. El análisis de semejanza toma como básicos los hechos de semejanza y trata de derivar de estos hechos la identidad de tipos y los hechos de ejemplificación. La idea es que los hechos de semejanza permiten explicar que dos particulares compartan una propiedad e, incluso, que cierto particular ejemplifique una propiedad. Compartir una propiedad consiste en asemejarse, y ejemplificar una propiedad consiste en mantener cierta estructura de semejanzas (tal vez con otros particulares fijados). Esta idea central del análisis de semejanza suele combinarse con el análisis de clases para obtener una versión sofisticada de este último. Se trata de seleccionar las clases que corresponden a propiedades genuinas en términos de clases de particulares relacionados por la relación de semejanza, tal vez mediante ciertos particulares fijados.

De hecho, sin embargo, no es difícil advertir que el análisis se semejanza no parte estrictamente del hecho de semejanza, sino de algo próximo al mismo, aunque ontológicamente más complejo. Puesto que con ellos se pretende explicar los hechos de ejemplificación, los hechos básicos de semejanza no pueden consistir meramente en dos particulares relacionados por la relación de semejanza, porque ello no bastaría para discriminar propiedades distintas. Por un lado, se habrá advertido que en el esbozo inicial de propuesta de explicación de los hechos de ejemplificación en términos de los hechos de semejanza, ya avanzábamos la posibilidad de apelar a "ciertos particulares fijados" (denominados "paradigmas»); por otro lado, cabe también apelar a grados de semejanza. La razón es la siguiente. Supongamos que todos los Ps son Qs y que ningún particular que ejemplifica $\mathrm{P}$ o $\mathrm{Q}$ ejemplifica ninguna otra propiedad. En ese caso, la relación de semejanza no permite aislar los Ps de los Qs, ni tan 
siquiera con la ayuda de particulares fijados o paradigmas: los Ps se asemejan entre sí, pero también se asemeja cualquier $\mathrm{P}$ con cualquier $\mathrm{Q}$, ya que, por hipótesis cualquier $\mathrm{P}$ es $\mathrm{Q}$. A continuación, examinaremos dos posibles respuestas a este problema que surgen de modo natural al examinarlo.

La primera de estas respuestas consiste en sostener que los Ps se asemejan en un aspecto distinto de aquél en que se asemejan los Qs, y que esta diferencia de aspectos de semejanza es la que constituye la diferencia entre los Ps y los Qs (entre la propiedad P y la propiedad Q, en definitiva). Por consiguiente, al análisis de semejanza debería incorporar aspectos de semejanza y postular que dos particulares comparten una propiedad P si se asemejan en cierto aspecto. Así, por ejemplo, se dirá que dos esmeraldas comparten la propiedad de ser verde si se asemejan en cierto aspecto. Si tenemos en cuenta que tendemos a referirnos de modo natural a este aspecto que permite identificar la propiedad de ser verde mediante el término «verdor» (y, así, decimos que dos esmeraldas comparten la propiedad de ser verde si se asemejan en el aspecto verdor), entonces se pone de manifiesto un dificil problema para este intento de refinamiento del análisis de semejanza: el problema de cómo explicar esta noción de aspecto de semejanza sin apelar a propiedades.

La segunda respuesta pasa por asumir que la relación de semejanza admite grados, es decir, que existen grados de semejanza. Convenimos en aceptar que los Ps se asemejan entre sí y también que los Qs se asemejan entre sí y que, puesto que todos los Ps son Qs, los Ps también se asemejan a los Qs. Sin embargo, los Qs que no sean Ps no se asemejarán entre sí tanto como los Ps se asemejan entre sí: el grado de semejanza entre los Ps es mayor que entre los Qs que no son Ps. Esta maniobra permite discriminar los Ps de los Qs.

Sin embargo, cabe señalar que el recurso de los grados de semejanza no permite dispensarnos sin más de los paradigmas, ni tan siquiera para poder explicar la identidad de tipos. Observemos que el hecho de que dos particulares tengan la propiedad $\mathrm{F}$ no puede consistir meramente en que estos particulares se asemejen, pues dos Gs también se asemejarán entre sí sin que para ello se requiera que sean F. Sin embargo, la inclusión de grados de semejanza todavía no es suficiente para resolver el problema, pues si decimos que dos particulares tienen la propiedad F si se asemejan en cierto grado, nada garantiza que no estemos todavía confundiendo los Fs con los Gs si se da el caso de que los Gs se asemejan entre sí en el mismo grado en que se asemejan los Fs. Nótese que no parece haber ninguna garantía de que propiedades distintas determinen necesariamente grados de semejanza distintos. 
No obstante, estas últimas consideraciones no deben inducir a pensar que no se puede formular un análisis de semejanza que no apele a paradigmas y que se halle libre de los problemas que acabamos de mencionar. De hecho, en el curso de este artículo discutiremos dos tipos de análisis de semejanza. Las teorías del primer tipo, como la que presentaremos a continuación, formulada por Price, emplea en el análisis paradigmas y grados de semejanza, mientras que las teorías del segundo tipo hacen uso tan sólo de grados de semejanza. Como veremos, existen diversas propuestas que se pueden incluir en el segundo tipo de teoría, todas las cuales parten del concepto de circulo de semejanza de Carnap.

\section{Paradigmas}

Veamos, en primer lugar, una teoría de la semejanza que apela a paradigmas. Con la ayuda de grados de semejanza y de paradigmas, se puede formular una primera versión del análisis del hecho de ejemplificación en los siguientes términos:

(A1) a tiene la propiedad P si y sólo si a tiene cierto grado de semejanza con cierto $\mathrm{P}$ paradigmático.

Este análisis permite explicar las propiedades como clases formadas en torno a estos paradigmas a partir de la relación de semejanza. Las versiones usuales del análisis de semejanza con paradigmas suelen partir de una colección finita, aunque plural, de paradigmas. Consideremos, por ejemplo, una versión de la idea de Price ${ }^{1}$ :

(A1') a tiene la propiedad P si y sólo si el grado de semejanza que a tiene con cada P-paradigma es mayor o igual que el grado de semejanza que existe entre los P-paradigmas ${ }^{2}$.

1 PRICE, 1953 p. 114.

2 Observemos que no bastaría con establecer que un objeto verde es un objeto que se asemeja a uno de los paradigmas al menos tanto como los paradigmas se asemejan entre sí. El problema es que un objeto no verde puede satisfacer dicha condición si se asemeja a uno de los paradigmas en un aspecto distinto del color, al menos tanto como los paradigmas se asemejan entre sí. Debemos tener en consideración que los paradigmas, con toda probabilidad, diferirán entre sí ampliamente en otros aspectos _ en forma, pongamos por caso-y que podemos hallar, por 
Sin embargo, este análisis presenta diversos problemas. A continuación discutiremos las objeciones que Armstrong ha dirigido a esta propuesta de Price.

(i) Sobredeterminación de paradigmas. Armstrong expone con gran claridad lo que entiende como un problema generado por el análisis de Price:

Es claramente posible que los paradigmas o ejemplares para una clase de propiedades sirvan como ejemplares para otra. Supongamos, por ejemplo, que los paradigmas para la clase de las cosas rojas son objetos que tienen una densidad muy alta. Los paradigmas seleccionarán la clase de cosas que son rojas o muy densas. Y, por tanto, no se seguirá que un objeto que se asemeja a los paradigmas para la clase roja, al menos tanto como los paradigmas se asemejan entre sí, es un objeto rojo. Porque podría ser denso y no verde ${ }^{3}$.

Un objeto denso, pero no rojo, no se asemejaría tanto a los paradigmas para la clase roja como los paradigmas se asemejan entre sí, ya que por hipótesis todos los paradigmas para la clase roja son rojos y densos. El hecho es que si dos propiedades coincidiesen en los paradigmas que determinan las clases respectivas, entonces el resultado no sería, contra lo que Armstrong pretende, que los paradigmas seleccionarían la unión de las clases originales (la clase de los particulares que son, o bien rojos, o bien densos), sino su intersección (la clase de los particulares que son rojos y densos a la vez), o, tal vez, más bien una subclase propia de esta intersección. Resulta evidente que, por otro lado, esta conclusión tampoco resulta en absoluto satisfactoria para el defensor de (A1'), pero depende del supuesto crucial de que las propiedades tengan paradigmas coincidentes.

Armstrong entiende que el problema que intenta presentar está estrechamente emparentado con el problema clásico de la coextensión puesto por Goodman al análisis de Carnap que, como hemos avanzado, no postula paradigmas. Sin que vayamos a introducir ahora el problema de la coextensión que veremos más adelante, cabe señalar que el análisis de Carnap no permite distinguir dos propiedades coextensivas cualesquiera. Es obvio que esto no es así

ejemplo, un objeto con exactamente la misma forma que uno de los paradigmas pero distinto en color. Ahora bien, es perfectamente posible que un objeto como el descrito se asemeje más a uno de los paradigmas de lo que los paradigmas se asemejan entre sí.

3 ARMSTRONG 1978, I, p. 48, énfasis mío. "Ejemplares" es la expresión que Price emplea para referirse a los paradigmas. 
en el caso del análisis de Price, pues dos propiedades pueden ser coextensivas sin ser confundidas por (Al'), bajo el supuesto de que existan paradigmas distintos para ambas propiedades, que permitan distintas semejanzas entre los particulares que ejemplifican ambas propiedades y los paradigmas que las caracterizan ${ }^{4}$. Sin embargo, es fácil adaptar el problema de la coextensión para presentarlo contraponerlo al análisis de Price: si dos propiedades son coextensivas y tienen paradigmas coincidentes, entonces el análisis comporta que se trata de una sola propiedad. Aunque la objeción es adaptable, no nos resulta obvio que tenga la misma fuerza que la que tiene contra las teorías de inspiración carnapiana, esencialmente porque necesita de la premisa añadida de los paradigmas coincidentes.

(ii) Independencia. Por otra parte, Armstrong defiende la idea de que la semejanza con paradigmas no determina propiedades 5 . De acuerdo con la versión del análisis de semejanza que estamos considerando, es constitutivo de tener una propiedad estar en ciertas relaciones de semejanza con otros objetos, los paradigmas. Sin embargo, sostiene Armstrong, esta idea es implausible, ya que, por el contrario, parece que debe haber cierto fundamento en los objetos que posibilite estas relaciones. El orden natural de la explicación es, precisamente, el inverso del que pretende el análisis de semejanza: es el hecho de que un objeto tenga una propiedad lo que explica las relaciones de semejanza que este objeto puede mantener con otros objetos. No obstante, no pensamos que estas consideraciones puedan constituir una objeción genuina contra el análisis de semejanza, pues no parece ser más que una mera petición de principio.

A pesar de ello, Armstrong intenta presentar un argumento que permita establecer que la ejemplificación de una propiedad no puede depender de la existencia de estas relaciones de semejanza entre sus ejemplos y los paradigmas ${ }^{6}$. El argumento parte del supuesto de que es conceptualmente posible que una propiedad tenga tan sólo un ejemplo. Consideremos un mundo posible en el que existe tan sólo una cosa verde —una esmeralda-, pongamos. ¿Qué perspectivas ofrece el análisis de semejanza que apela a paradigmas de explicar el hecho de que la esmeralda sea verde en sus propios términos? Armstrong le concede dos posibilidades alternativas:

Véase ARMSTRONG 1978, I, p. 48.

ARMSTRONG 1978, I, pp. 50-53.

6 ARMSTRONG 1978, pp. 52-53. 
(a) La primera opción pasa por sostener que las partes espaciales y las fases temporales propias de la esmeralda verde se asemejan entre sí, y afirmar que el color verde de la esmeralda en su totalidad depende de las relaciones de semejanza entre estas partes y fases verdes.

(b) La segunda posibilidad consiste en afirmar que la totalidad de la esmeralda verde se asemeja a sus partes y fases.

Consideremos la primera opción, que presenta una dificultad fundamental. El problema original que se había planteado para la esmeralda verde se puede replantear ahora para sus partes y fases (y después para sus sub-partes y sub-fases, $y$ así indefinidamente), ya que parece plausible pensar que cualquiera de estas partes y fases podría haber sido el único objeto del mundo, sin que esto hubiera afectado a su color verde. De nuevo, surge la pregunta natural de cómo explicar el color verde estas partes o fases. Ahora, o bien se alcanza un estadio en el que hallamos átomos verdes, de forma que su color verde no depende de semejanza alguna, cosa que es contraria al análisis de semejanza, o bien el proceso se repite indefinidamente sin que se alcance jamás ningún objeto verde cuyo color dependa de semejanzas entre este objeto y otros objetos.

Armstrong entiende que ambas posibilidades son incompatibles con la versión del análisis de semejanza que estamos discutiendo. Consideremos en primer lugar la posibilidad relativa a los átomos verdes. ¿Qué relación de semejanza puede garantizar el color verde de un átomo (esto es, un punto espacio temporal) solitario? Tan sólo parece que quede abierta la opción de afirmar que los que constituye el color del átomo es que se asemeja a sí mismo. Sin embargo, esta respuesta genera, a juicio de Armstrong, dos graves problemas.

Por un lado, Armstrong cree que la última afirmación contraviene el espíritu del análisis de semejanza, que mantiene que la ejemplificación de una propiedad por parte de un particular depende de las relaciones que este particular tiene con otros particulares. Por el contrario, en el caso del átomo, no existen "otros" particulares. Sin embargo, tal vez Armstrong se excede en sus conclusiones. Quizás podamos concederle que la afirmación en cuestión contraviene el espíritu del análisis de semejanza, pero no la letra del análisis de Price, pues en ningún momento nos hace explícito que los paradigmas deban ser distintos del particular que tiene la propiedad. En cualquier caso, cabe decir también que el espíritu del análisis debe entenderse como dirigido a los casos normales, y no a casos límite como los que presenta Armstrong involucrando propiedades con un solo ejemplo. 
En segundo lugar, Armstrong rechaza en general la posibilidad de relaciones reflexivas, esencialmente por dos razones: son determinables a priori y no confieren poderes causales a los particulares que los ejemplifican ${ }^{7}$. El propio Armstrong reconoce la existencia de algunos casos aparentes de relaciones reflexivas que parecen constituir contraejemplos a sus objeciones, es decir, que no son aparentemente determinables a priori y otorgan poderes causales a los particulares que las ejemplifican, y a continuación trata de mostrar que se trata de casos espúreos de relaciones reflexivas. Los resultados no son, a nuestro juicio, concluyentes. En resumen, no es evidente qué fuerza pueden tener estas razones que permiten establecer que el caso del átomo verde aislado resulta intratable para la versión de Price del análisis de semejanza.

Por otro lado, tampoco es claro a nuestro juicio que la insatisfacción de Armstrong respecto al segundo extremo del dilema concerniente al caso a) esté justificada. Lo que parece insatisfactorio con la cadena infinita descendente de partes y fases que se genera no parece residir tanto en el hecho de que no se hallen átomos que no satisfagan la letra del análisis de semejanza, como en el hecho de que no se hallen átomos de ningún tipo, y que, consiguientemente, la explicación prometida del color verde de nuestra esmeralda queda, por así decirlo, suspendida en el vacío. Aunque no se hallen átomos que satisfagan la letra del análisis de semejanza, el espíritu sí se mantiene. Debemos conceder que es cierto que se está afirmando que el color de la esmeralda no depende de las relaciones de semejanza que la propia esmeralda mantiene con otros objetos, en contra de lo que postulaba la letra original del análisis de semejanza. Sin embargo, tal vez consideraremos que se trata de un problema menor si pensamos que, en definitiva, el color de la esmeralda vendrá determinado por las relaciones de semejanza que involucran cosas muy próximas a la esmeralda, si bien no estrictamente idénticas con la misma: sus partes propias, entidades parcialmente idénticas a la esmeralda. Sea como fuere, no parece que el segundo de los extremos del dilema lleve a buen puerto. Como ya hemos dicho, las razones de Armstrong contra el primer extremo del dilema no nos parecen concluyentes, aunque aportan ciertas dudas serias acerca de su plausibilidad.

7 ARMSTRONG 1978, II, pp. 92-93. Recientemente, sin embargo, parace haber cambiado de parecer; en Armstrong 1997 se pronuncia a favor de la relación de semejanza exacta, que es reflexiva. Véase Armstrong 1997, p. 23. 
Consideremos ahora la segunda alternativa, $b$ ), que pasa por sostener que la totalidad de la esmeralda verde se asemeja a sus partes y fases. Armstrong entiende que que existen dos tesis distintas que surgen de la anterior en función de si entendemos "asemejarse a sus partes y fases" como b.1): asemejarse a la totalidad de sus partes o fases; o como b.2): asemejarse a las partes y fases individualmente. Sin embargo, como ya hemos visto, de acuerdo con Armstrong, la primera salida, b.1), hace absurdo el propósito inicial del nominalista de semejanza de fundamentar la ejemplificación de una propiedad por parte de un particular en hechos de semejanza relativos a este particular y a otros particulares diferentes del mismo, ya que, en definitiva, deberá admitir que en algunos casos basta con las relaciones de semejanza que el particular en cuestión tiene consigo mismo. Sin embargo, si entendemos la segunda alternativa en el sentido especificado en b.2), no hacemos sino posponer el problema. Supongamos, por mor de la simplicidad, que el hecho de semejanza pertinente es que la esmeralda, toda ella, se asemeja a cierta parte propia suya, $p$. Ahora bien, que la esmeralda se asemeje a $p$ puede entenderse de dos maneras: que la "esmeralda-menos- $p$ " se asemeje a $p$, en cuyo caso vamos al caso a), ya descartado, o bien debemos decir que es toda la esmeralda, incluyendo a $p$, que se asemeja a $p$, en cuyo caso la ejemplificación de la propiedad volvería a depender de la semejanza de $p$ consigo misma.

\section{Círculos de semejanza}

Como hemos avanzado con anterioridad a la discusión de la teoría de Price, no todo análisis de semejanza apela a paradigmas. El referente clásico de ciertas versiones del análisis de semejanza que no emplean paradigmas es el concepto de círculo de semejanza ideado por Carnap, que aparece en su libro de 1928, Der Logische Aufbau der Welt ${ }^{8}$. Estrictamente, Carnap no pretende construir propiedades, sino meramente cualidades, elementos de la experiencia sensible del sujeto, pero su idea es extrapolable a una teoría de las propiedades como entidades objetivas. La clase que Carnap identificará con una cualidad, el círculo de semejanza, viene determinada por la siguiente condición: cada miembro de la clase se asemeja más a cualquier otro miembro de la clase

8 CaRnaP $1967, \$ \$ 67-93,108-120$. 
de lo que cualquiera de ellos se asemeja a cualquier otro objeto que no pertenece a la clase. Como puede observarse, lo que determina la pertenencia a la clase son las relaciones de semejanza que sus miembros establecen entre sí y con los objetos que están fuera de la clase. Descrita en términos de ejemplificaciones de propiedades, la condición de Carnap afirmaría que que el hecho de que un particular ejemplifique una propiedad, $P$, consiste en que el particular en cuestión se asemeje más a los otros particulares que ejemplifican la propiedad de lo que cualquier otro de estos particulares se asemeja a cualquier otro objeto que no la ejemplifique, de forma que la condición está dentro de la línea general que inspira a los análisis de semejanza. Las palabras en cursiva ponen de manifiesto que la anterior descripción de la condición de inspiración carnapiana no puede constituir una definición estricta de ejemplificar una propiedad porque sería circular. Más bien la condición debe entenderse como la restricción que explica cómo los particulares se agrupan en propiedades, y lo hacen de manera colectiva, holista. Si se prefiere, se puede repensar el análisis de semejanza como un refinamiento del nominalismo de clases que permite discriminar, entre el dominio de todas las clases de particulares, aquellas clases que constituyen genuinas propiedades y las que no. Así, sólo las clases cuyos miembros satisfaciesen la condición carnapiana serían clases naturales. Podemos formalizar la condición carnapiana como sigue:

(C) $\mathrm{P}$ es una clase natural si y sólo si $\forall x, y, z[(x, y \notin \mathrm{P} \wedge z \in \mathrm{P} \rightarrow(\mathrm{S}(x, y)$ $>S(y, z))]$

(donde "S(a,b)» indica el grado de semejanza entre $a$ y b.)

Una de las primeras ventajas de (C) respecto a la versión con paradigmas es que ahora no es válida la objeción de Armstrong, que pretendía mostrar la independencia del hecho de que un particular ejemplifique una propiedad del hecho de que dicho particular esté en ciertas relaciones de semejanza con unos paradigmas, como pretendía el análisis de Price; a saber, la posibilidad de concebir el particular aislado ejemplificando la propiedad, sin otros particulares del mismo tipo que pudiesen ejercer de paradigmas. Con el objeto de simplificar la discusión, pongamos que estamos considerando un átomo verde en un mundo posible, solitario en su clase unitaria de cosas verdes de ese mundo posible considerado. Con independencia de la opinión que nos merezcan las razones de Armstrong para oponerse a la existencia de las relaciones reflexivas, parece que estas razones no pueden ser suficientes para inducirnos a pensar que existe un objeto no verde en aquel mundo, al cual el átomo se asemeja más que 
a sí mismo (que es el único objeto verde en aquel mundo, por hipótesis), justamente lo que debería suceder para que no pudiéramos pensar, a la luz de la condición $(\mathrm{C})$, que el átomo es verde en aquel mundo.

Sin embargo, como es bien sabido, el análisis de Carnap no está exento de problemas. Goodman presenta dos objeciones centrales a la propuesta de Carnap, conocidas respectivamente como el problema de la coextensión y el problema de la comunidad imperfecta ${ }^{9}$.

El problema de la coextensión ya había aparecido cuando nos ocupábamos del nominalismo de clases, y ahora continua vigente a pesar del nuevo criterio propuesto para distinguir las clases naturales, esto es, las clases que son la extensión de las propiedades genuinas. Este análisis de inspiración carnapiana no permite distinguir dos propiedades que comparten la misma extensión. Para poder distinguir dos propiedades en términos de (C), es necesario que los ejemplos de las dos propiedades formen dos clases naturales distintas, pero para ello, por supuesto, es necesario que formen dos clases distintas, lo que no es el caso. Para resolver este problema resulta necesario apelar a particulares meramente posible, possibilia. En seguida veremos que otras dificultades del análisis conducen también de modo natural a postular este elemento modal.

Por lo que respecta al problema de la comunidad imperfecta, Quine realiza una presentación breve e interesante del mismo:

Una tentativa distinta, adaptada de Carnap, es la siguiente: un conjunto es un género si todos sus miembros se asemejan más entre sí de lo que todos ellos se asemejan a cualquier cosa fuera de la clase. En otras palabras, cada no-miembro difiere más de algún miembro de lo que aquel miembro difiere de cualquier otro miembro. Sin embargo, como Goodman mostró en una crítica a Carnap, esta construcción cede por lo que Goodman denominó el problema de la comunidad imperfecta. Así, consideremos el conjunto de todas las cosas rojas y redondas, rojas y de madera, o redondas y de madera. Cada miembro de este conjunto se asemeja más de alguna manera a cualquier otro miembro; cuando menos, en ser rojo, o en ser redondo, o en ser de madera, y tal vez en dos o tres de estos aspectos u otros. Además, es concebible que no exista nada fuera del conjunto que se asemeje a todo miembro del conjunto ni tan siquiera en el menor de estos grados. El conjunto,

9 Goodman 1966, cap. V, $\$$ III. 
pues, satisface la definición propuesta de género. Sin embargo, a buen seguro que no se trata de lo que se entiende por género. Admite pelotas de críquet amarillas y pelotas de goma rojas, excluyendo pelotas de goma amarillas ${ }^{10}$.

En este fragmento, Quine trata como equivalentes la propuesta de Carnap que hemos formalizado por medio de $(\mathrm{C})$ y lo que puede ser formalizado en los siguientes términos:

(Q) $\mathrm{P}$ es una clase natural si y sólo si $\forall x[x \notin \mathrm{P} \wedge \exists \mathrm{y}(\mathrm{y} \in \mathrm{P} \wedge] \forall z(z \in \mathrm{P}$ $\rightarrow \mathrm{D}(\mathrm{x}, \mathrm{y})>\mathrm{D}(\mathrm{y}, \mathrm{z})))]$

(donde « $\mathrm{D}(a, b)$ " expresa el grado de diferencia existente entre dos particulares, $a$ y $b$ )

De hecho, sin embargo, (C) y (Q) no son equivalentes. Eli Hirsch propone un contraejemplo para (C) que no afecta a (Q) ${ }^{11}$. El contraejemplo muestra que la satisfacción de la condición de Carnap no es condición necesaria para que una clase sea natural. Consideremos dos propiedades, $\mathrm{P}$ y $\mathrm{Q}$, compatibles entre sí, y tres particulares, $x, y$ y $z$, tales que $x$ tiene $\mathrm{P}$ y Q, $y$ tiene $\mathrm{P}$, pero no $\mathrm{Q}$, y $z$ tiene $\mathrm{Q}$ pero no $\mathrm{P}$. En apariencia, P y $\mathrm{Q}$ pueden ser ambas perfectamente naturales, pero la condición de Carnap no resulta satisfecha, ya que si $S(x, y)>$ $\mathrm{S}(x, z)$, entonces, de acuerdo con (C), $\mathrm{Q}$ no es natural; mientras que si no es cierto que $S(x, y)>S(x, z)$, entonces, de acuerdo con (C), $\mathrm{P}$ no es natural.

Dejando al margen estas consideraciones, hay una cuestión de especial importancia para la presente discusión, que afecta tanto a (C) como a (Q). Aun soslayando otros problemas que estos análisis puedan presentar, para que $(C)$ y (Q) resulten, en principio, mínimamente plausibles, y a pesar de las intenciones iniciales de sus proponentes, deben entenderse cuantificando sobre possibilia. Por ejemplo, consideremos (C). Supongamos que $\mathrm{P}$ es una propiedad genuina y que $a$ no es $P$. ¿Qué nos garantiza que podamos hallar ni tan siquiera un $\mathrm{P}$, pongamos $b$, que se asemeje más a cualquier $\mathrm{P}$ de lo que se asemejan $a$ y $b$ ? ¿ Por qué no podría darse el caso de que $a$ y $b$, a pesar de no tener la propiedad $P$ en común, compartiesen toda una serie de propiedades, $Q 1, \ldots, Q n$, que ningún otro $P$ tuviera, de forma que el hecho de compartir estas propiedades ajenas a los otros Ps garantizara que, al fin y al cabo, la diferencia entre $a$ y

10 QUINE 1969, pp. 121-122.

11 HiRSCH 1993, pp. 56-57. 
$b$ no fuera mayor que entre $b$ y los otros Ps? Nos parece obvio que sólo si el dominio de cuantificación incluye possibilia podemos tener alguna garantía de ello. De ahora en adelante, entenderemos que tanto en los dos análisis anteriores como en algunas nuevas propuestas que consideraremos se están presuponiendo entidades modales en el dominio de cuantificación; un supuesto que, por otra otra parte, también era exigido para dar cuenta del problema de la coextensión ${ }^{12}$.

Aunque el contraejemplo anterior no afecta a (Q), el propio Quine piensa que la condición de (Q) es satisfecha por una clase que constituye una "comunidad imperfecta", sin que la clase en cuestión tenga por qué ser natural. Consideremos tres propiedades, P, Q y R, y definamos la clase C por medio de:

Para todo $x, x$ ( C si y sólo si $x$ tiene al menos dos de las tres propiedades: P, Q, R.

Desde el punto de vista intuitivo, $\mathrm{C}$ no tiene por qué ser una clase natural, es decir, no tiene por qué darse el caso de que todos los objetos que pertenezcan a $C$ compartan alguna propiedad. Sin embargo, Quine aplica la idea de Goodman a su versión del análisis de Carnap argumentando que si tomamos cualquier particular fuera de $\mathrm{C}$, siempre podemos elegir un particular en $\mathrm{C}$ de forma que la diferencia entre el primero y el segundo sea mayor que la que pueda haber entre el segundo y cualquier otro particular en $C$. Sea $a$ el individuo fuera de C. Así, a tiene, a lo sumo, tan sólo una de las propiedades $\mathrm{P}, \mathrm{Q}$, $R$. Supongamos que tiene $P$, y observemos que si a no tuviera ninguna de las tres propiedades mencionadas, el argumento no se vería afectado sustancialmente. Tomemos ahora un $\mathrm{C}$, digamos $b$, cuyas diferencias con $a$ sean las mayores posibles en relación a las tres propiedades en juego; es decir, $b$ tiene Q y R pero no P. Ahora bien, resulta que el grado de diferencia entre $b$ y cualquier otro $\mathrm{C}$ será menor que la que existe entre $a$ y $b$. La razón es que cualquier otro $\mathrm{C}$ tendrá en común con $b$ al menos una de las propiedades $\mathrm{P}, \mathrm{Q}, \mathrm{R}$, como sucede siempre entre dos elementos cualesquiera de C.

Cabe señalar que en este argumento juega un papel crucial el hecho de que en la condición que Quine emplea para definir la clase C se está cuantificando

12 Nótese, sin embargo, que el postulado de possibilia contraviene el espíritu nominalista de la propuesta del análisis de semejanza. De hecho, autores como Quine rehúyen con firmeza el postulado de este tipo de entidades modales. 
sobre possibilia. Debemos pensar en qué garantía tenemos de poder especificar $b$ del modo en que lo hemos hecho. En caso contrario, ¿qué nos garantizaría la existencia de un $\mathrm{C}$ que no compartiera con a ninguna de las propiedades que determinan la comunidad imperfecta? Resulta destacable que ni Quine ni Hirsch hagan explícito este supuesto que, como hemos visto, resulta esencial para dotar de un mínimo sentido a (Q) e, irónicamente, también para presentar la objeción de la comunidad imperfecta.

Como indica Hirsch, el anterior ejemplo de comunidad imperfecta es un caso particular de toda una familia de casos que pueden construirse a partir de la siguiente idea. Si partimos de una serie finita de propiedades, $\mathrm{P}_{1}, \ldots \mathrm{P}_{n}$, se puede construir la clase no natural que satisface el criterio expresado en (Q), definiéndola como la clase de objetos que tienen al menos $m$ de las $n$ propiedades en cuestión, donde, naturalmente, $m<n$, pero $n<2 m^{13}$.

A continuación, veremos cómo la propuesta de Quine plasmada en (Q) tiene, además, el problema de no excluir las propiedades disyuntivas. Después, comentaremos una propuesta de Hirsch en la línea de Quine que evita el problema de las propiedades disyuntivas, así como la objeción de Hirsch contra la versión de Quine del argumento goodmaniano de la comunidad imperfecta. Finalmente, consideraremos un nuevo análisis de la noción de clase natural propuesto por Hirsch, y veremos los dos grandes problemas que debe afrontar: el problema del fragmento del continuo y el problema de la implicación conjuntiva.

Hirsch apunta una dificultad añadida al problema de la comunidad imperfecta, que atribuye a la versión de Quine del análisis de Carnap: la unión de dos clases naturales cualesquiera satisface la condición puesta por Quine y, por consiguiente, de acuerdo con (Q), resulta ser también una clase natural ${ }^{14}$. Sean $P$ y $Q$ dos propiedades cualesquiera y consideremos el conjunto $U$ formado por los objetos que tienen la propiedad P o la propiedad Q. Notemos que, incluído en $\mathrm{U}$, hallamos el conjunto I de los objetos que tienen ambas propiedades, $\mathrm{P}$ y Q. Observemos también que cualquier objeto en compartirá con cualquier objeto en $\mathrm{U}$ una propiedad al menos - dos propiedades al menos, si el objeto de $U$ también está en $I$. Si ahora consideramos un objeto $x$

13 La pseudo-propiedad en cuestión sería tener más de la mitad de las propiedades genuinas.

14 HiRsCH 1993, p. 209. 
cualquiera fuera de $\mathrm{U}$, y tomamos un objeto, $y$, en I, que difiera de $x$ en todas sus propiedades, resultará que el grado de diferencia entre $x$ e $y$ será mayor que el grado de diferencia existente entre $y$ y cualquier otro elemento de $\mathrm{U}$, con el cual compartirá, al menos, $\mathrm{P} \circ \mathrm{Q}^{15}$.

El siguiente análisis, propuesto por Hirsch, evita este último problema de las propiedades disyuntivas, aunque coincide con (Q) en asimilar las estructuras de comunidad imperfecta a clases naturales:

(Q') $\mathrm{P}$ es una clase natural si y sólo si $\forall x[x \notin \mathrm{P} \rightarrow \exists y(\mathrm{y} \in \mathrm{P} \wedge \forall z, u$ $((z \in \mathrm{P} \wedge u \in \mathrm{P}) \rightarrow \mathrm{D}(\mathrm{x}, \mathrm{y})>\mathrm{D}(\mathrm{z}, \mathrm{u})))]$

Como hemos visto, para que la objeción de la comunidad imperfecta pueda formularse se requiere el supuesto de que el análisis de semejanza cuestionado establece una condición en la que se cuantifica sobre possibilia. También hemos observado cómo es difícil que ello permita concebir una réplica a la objeción, porque se trata de un supuesto que también es necesario para que el análisis resulte mínimamente razonable. Sin embargo, Hirsch pone de manifiesto otro supuesto presente de manera implícita en el argumento quineano que sí parece constituir una base firme para responder a la objeción ${ }^{16}$.

Para tratar de ver cuál es la naturaleza del supuesto que Hirsch apunta, revisaremos el argumento anterior que pretendía establecer que cualquier estructura de comunidad imperfecta satisface la condición expresada en (Q'). Ahora, sin embargo, añadiremos a los anteriores supuestos que las propiedades $Q$ y $R$ que $a$ no tiene son ser rojo y ser redondo. $Y$ consideremos la afirmación final del argumento que sostiene que cualquier otro $\mathrm{C}$, distinto de $a$ y $b$, digamos $c$, será tal que el grado de diferencia que existe entre $a$ y $b$ será mayor que el existente entre $b$ y $c$, ya que compartirán al menos una de las propiedades $P$, ser rojo y ser redondo, mientras que $a$ y $b$ no comparten ninguna. La cuestión es que esta última afirmación carece de fundamento en el caso de que $a$, a pesar de no ser rojo ni redondo, sea casi rojo y redondo. Se puede concebir a a dotado de un tono de naranja muy próximo al rojo y de una forma ovalada prácticamente redonda. En ese caso, no diríamos que la diferencia entre $a$ y $b$ es mayor que la que existe entre

15 Como se habrá observado, en este argumento se está presuponiendo que en el análisis (Q) se cuantifica sobre possibilia; es por ello que queda garantizada la existencia de un $U$ con los rasgos que hemos atribuido a $y$.

16 HiRSCH 1993, p. 214. 
by $c$. Así pues, en el argumento anterior se había estado presuponiendo que, una vez fijados ay las propiedades que $a$ no tiene del dominio de las propiedades que constituyen la estructura de la comunidad imperfecta, se podría fijar un $\mathrm{C}$ que no solamente tenía las propiedades de la comunidad que $a$ no tenía, Q y R (y no tenía las que $a$ tenía) sino que, además, el $C$ en cuestión podía ser tan distinto de a como fuera conveniente en relación a estas propiedades; en particular, suficientemente distinto como para que el grado de diferencia entre ambos fuese mayor que entre el $\mathrm{C}$ en cuestión y cualquier otro $\mathrm{C}$.

Estas consideraciones permiten establecer que la formulación del problema de la comunidad imperfecta en los términos propuestos por Quine es incorrecta. Para poder construir una versión adecuada del problema, es necesario hacer explícito el supuesto relativo a cada una de las tres porpiedades P, Q y R: ningún objeto que no tiene alguna de ellas puede ser relativamente semejante a ningún objeto que la tiene ${ }^{17}$.

Al margen de este problema de la comunidad imperfecta, Quine menciona otro problema asociado que afecta también a la propuesta de análisis de inspiración carnapiana en términos de semejanza, en cualquiera de las versiones aquí presentadas. Hirsch lo denomina el problema de las esferas: la idea es que ciertos conjuntos, denominados esferas de semejanza, satisfacen la condición de semejanza pero no determinan propiedades genuínas. Una esfera de semejanza, Ex,y, determinada por ciertos objetos fijados, $x$ e $y$, se define como sigue:

$z \in \mathrm{E}_{x, y}$ si y sólo si $S(x, y) \leq \mathrm{S}(x, z)$.

(Se entiende que los zs varían sobre un dominio de possibilia, pero que $x \mathrm{e}$ $y$ son tomados con sus rasgos actuales, a efectos de semejanza.)

Una esfera de semejanza en torno de cierto objeto está formada por todos los objetos que se asemejan a este objeto en un grado mínimo fijado. El mínimo se fija como el grado de semejanza entre el objeto, $x$, en torno del cual se genera la esfera, y cierto objeto $y$.

Intuitivamente, una esfera de semejanza no tiene por qué constituir una clase natural; sin embargo, cualquier esfera de semejnaza satisface el criterio de

17 Como indica Hirsch, todavía habría que añadir algún supuesto más, como que todas las tres propiedades deben ser mútuamente compatibles y que ninguna conjunción de cualesquiera dos de esas propiedades implica la tercera. Véase Hirsch 1993, p. 215. 
semejanza. Hirsch desarrolla un argumento que establece que cualquier esfera de semejanza satisfce (Q') ${ }^{18}$.

Para acabar con esta revisión de propuestas de análisis de semejanza de inspiración carnapiana y los problemas que llevan asociados, comentaremos la propuesta reciente de Hirsch que permite salvar el problema de la comunidad imperfecta y problemas afines como el problema de las esferas. La idea intuitiva que anima al análisis de Hirsch es que compartir propiedades aumenta la semejanza. Esta idea permite construir la noción de clase natural en los siguientes términos:

(H) $\mathrm{P}$ es una clase natural si y sólo $s i \forall x, y[(x \in \mathrm{P} \wedge y \notin \mathrm{P}) \wedge \exists z(z \in \mathrm{P} \wedge$ $\forall u((u \in \mathrm{P} \wedge \mathrm{S}(z, y) \leq \mathrm{S}(y, u)) \rightarrow \mathrm{S}(u, x)>\mathrm{S}(x, y))]$

En la definición, debemos entender que la parte de la condición relativa a $z$ cumple la función de determinar un umbral de semejanza con $y$, de forma que cualquier $\mathrm{P}$ que se asemeje a $y$ más de lo que $z$ se asemeje a $y$, deberá asemejarse más a $x$ que a $y$. La idea, por tanto, es que si consideramos un objeto en $\mathrm{P}$ y un objeto cualquiera fuera de $\mathrm{P}$, cualquier $\mathrm{P}$ que se asemeje al no $\mathrm{P}$ más de lo que el umbral establece deberá asemejarse más al primer $\mathrm{P}$ de lo que se asemejaba el no P.

Veamos, en primer lugar, como (H) salva el problema de la comunidad imperfecta. Tomemos de nuevo la clase $\mathrm{C}$. Consideremos un $\mathrm{C}$ cualquiera, digamos $x$, y supongamos, sin pérdida de generalidad, que $x$ tiene $\mathrm{P}$ y $\mathrm{Q}$, pero no tiene R. Sea $y$ tal que $y$ tiene $\mathrm{Q}$, pero no tiene ni $\mathrm{P}$ ni R. Así, y no está en C. Consideremos ahora un $\mathrm{C}$ tan semejante a $y$ como sea posible y que, además, sea $\mathrm{Q}$ y $\mathrm{R}$ pero no sea P. Llamémosle $u$. Ahora observemos que no tiene por qué darse el caso que $u$ se asemeje más a $x$ de lo que $x$ se asemeja a $y$, pues $u$ y $x$ se asemejan solamente en el aspecto relativo a $Q$, mientras que $x$ e $y$ se asemejan en los aspectos relativos a $Q$ y $R$.

A pesar de esta ventaja relativa respecto a los análisis alternativos anteriores, como el propio Hirsch admite, existen dos graves problemas que esta nueva propuesta debe afrontar. Por un lado, la condición es demasiado débil: admite clases que no constituyen ninguna clase natural. Por otro lado, es demasiado fuerte: excluye clases que pueden perfectamente constituir propiedades. Hirsch

18 Véase HirSCH 1993, pp. 216-217. 
ofrece un reflexión interesante acerca de la importancia relativa de estas dos objeciones: mientras que la segunda objeción es específica de su propuesta, existen buenas razones para pensar que las clases admitidas incorrectamente son indistinguibles de otras clases naturales en términos de relaciones de semejanza, de forma que si las ideas de Hirsch respecto a estas clases fuesen correctas, todavía existiría una objeción de alcance general contra el proyecto de analizar las propiedades en términos de relaciones de semejanza entre particulares.

Vamos primero con la objeción específica, que Hirsch denomina el problema de la implicación conjuntiva. Definimos primero la relación de implicación conjuntiva, entre pares de clases y clases: dos clases, $\mathrm{P}$ y $\mathrm{Q}$, implican conjuntivamente una clase, $\mathrm{R}$, si son mútuamente compatibles, independientes dos a dos, y P\&Q implica R. Decimos que tres clases, P, Q, R, se implican conjuntivamente si P y $Q$ implican conjuntivamente a R; Q y R implican conjuntivamente a P, y, finalmente, $\mathrm{P}$ y $\mathrm{R}$ implican conjuntivamente a $\mathrm{Q}$. El problema de la implicación conjuntiva es simplemente que las tríadas de clases que se implican conjuntivamente no tienen por qué satisfacer la condición expresada en (H). Supongamos, por ejemplo, que ser cerrado, tener cuatro lados, y tener cuatro ángulos son propiedades genuínas. Notemos que sus extensiones se implican conjuntivamente. Sea $x$ un cuadrado, es decir, un objeto con las tres propiedades anteriores. Sea $y$ un triángulo perforado (un triángulo al cual se le ha eliminado uno de los puntos de sus lados). Y sea $z$ un triángulo. Ahora, para que la propiedad de ser cerrado satisfaciera $(\mathrm{H})$ debería darse el caso de que $S(x, z)>S(x, y)$, mientras que para que la propiedad de tener cuatro lados satisfaciera $(\mathrm{H})$ debería darse el caso contrario de que $S(x, z)<S(x, y)$. Puesto que ambas condiciones no pueden darse al mismo tiempo, resulta que, de acuerdo con $(\mathrm{H})$, una de las dos clases correspondientes no puede ser natural. La solución de Hirsch a este problema pasa por considerar estos casos de implicación conjuntiva como serias excepciones a $(\mathrm{H})$ y trata de revisar el análisis incluyendo una cláusula de excepción que incorpora un expdiente recursivo:

$\left(\mathrm{H}^{\prime}\right) \mathrm{P}$ es una clase natural si y sólo si para todo $x, y$, si $x \in \mathrm{P}$ e $y \notin \mathrm{P}$, entonces
a) $\exists z(z \in \mathrm{P} \wedge \forall u((u \in \mathrm{P} \wedge \mathrm{S}(z, y) \leq \mathrm{S}(y, u)) \rightarrow \mathrm{S}(u, x)>\mathrm{S}(x, y))]$,

o bien

b) hay clases, $\mathrm{Q}$ y R, al menos una de las cuales es natural, tal que $x$ pertenece a ambas, $y$ a sólo a una de ellas, y P, Q y R se implican conjuntivamente. 
Las excepciones a la condición expresada en $(\mathrm{H})$ están restringidas a aquellas generadas por clases que se implican conjuntivamente y alguna de las cuales es natural ${ }^{19}$.

Todavía queda pendiente discutir la objeción de insuficiencia de la condición expresada en $(\mathrm{H})$, que Hirsch considera un grave problema para cualquier tentativa de análisis de las propiedades en términos de relaciones de semejanza entre particulares ${ }^{20}$. El problema se puede formular a partir de propiedades naturales con la misma estructura que los colores o las formas. Supongamos, por mor del argumento, que los colores son propiedades naturales. Así, ser rojo, ser naranja, o ser azul son propiedades naturales según nuestro supuesto. Fijemos ahora dos tonos determinados de rojo y naranja, rojor y naranjas, y consideremos la propiedad $\mathrm{P}^{*}$ de tener un color entre rojo $r$ y naranjas. Hirsch sostiene que $\mathrm{P}^{*}$ satisface la condición de semejanza expresada por $\left(\mathrm{H}^{\prime}\right)$, pero entiende que dicha propiedad no tiene por qué se natural.

Conviene puntualizar que Hirsch no ofrece ningún argumento directo que establezca que $\mathrm{P}^{*}$ satisface la condición de semejanza propuesta en $(\mathrm{H})$. La única razón que proporciona para justificar esta afirmación aparece en una nota a pie de página:

Estoy suponiendo que el color es un "fenómeno natural" (véase Kripke 1980 , p. 134), y entiendo que esto implica que una propiedad como Rojo es natural y satisface la condición $(\mathrm{N})[(\mathrm{H})]$. Se sigue entonces que $\mathrm{P}^{*}$ también satisface $(\mathrm{N})[(\mathrm{H})]$. Si los colores no fuesen considerados propiedades naturales, entonces habría que modificar mis ejemplos, pero el problema no variaría $^{21}$.

No parece, sin embargo, que estas consideraciones sean suficientes para establecer lo que Hirsch pretende. No vemos que el hecho de que supongamos que los colores son naturales garantice que $\mathrm{P}^{*}$ también lo es. Consideremos un objeto rojo que también tiene $\mathrm{P}^{*}$ en virtud de tener un tono muy próximo a rojor $y$ un objeto rojo que no es $\mathrm{P}^{*}$, pero que también tiene un tono de rojo

19 HiRSCH 1993, pp. 220-221.

20 Hay que señalar también que las propiedades negativas generan problemas de insuficiencia. La propuesta de Hirsch, $(\mathrm{H})$, consigue exluir negaciones de propiedades que satisfacen cierta condición compleja, pero no consigue excluirlas todas. Véase Hirsch 1993, pp. 216-219.

21 HirSCH 1993, p. 60, n. 18. 
próximo a rojor. Consideremos ahora un objeto naranja que también es $\mathrm{P}^{*}$ en virtud de tener un tono muy próximo a naranjas. No vemos razón alguna por la que el objeto rojo que es $\mathrm{P}^{*}$ deba asemejarse más al objeto naranja de lo que se asemeja al otro objeto rojo.

Tal vez Hirsch está pensando que si los colores son naturales, entonces $\mathrm{P}^{*}$ también debe ser natural, porque se trata de clases que determinan el mismo patrón de semejanzas entre particulares, puesto que se trata de propiedades estructuralmente idénticas. La cuestión, entonces, no es, contra lo que Hirsch sugiere en la nota, si lo colores son o no de hecho naturales, sino si cualquier propiedad, o estructura de propiedades, que determine el mismo patrón de estrcuturas de semejanza entre particulares que $\mathrm{P}^{*}$, puede ser una propiedad natural a la luz de su propio análisis $(\mathrm{H})$. Y parece que la conclusión es precisamente que no, si nuestras consideraciones contra la tesis de que $\mathrm{P}^{*}$ es natural son correctas.

La conclusión que, a nuestro juicio, hay que extraer de todo esto es que si se acepta el criterio impuesto por $(\mathrm{H})$, entonces no puede haber propiedades naturales que formen estructuras como la de los colores o la de las formas: series continuas de determinables. Por tanto, si alguien cree que existen buenas razones independientes para sostener que puede haber propiedades naturales de este tipo, entonces deberá pensar en abandonar $(H)$. Pero no solamente $(H)$, porque pensamos que Hirsch está en lo cierto cuando sostiene que propiedades como $\mathrm{P}^{*}$ resultan problemáticas para el proyecto de analizar las propiedades en términos de relaciones de semejanza entre particulares en su concepción global. Esta conclusión se sigue del hecho de que no parece haber ninguna manera no ad hoc de distinguir entre rojo y $\mathrm{P}^{*}$ meramente en términos de semejanza entre particulares. De hecho, propiedades con esta estructura violan el principio que anima al nominalismo de semejanza, a saber, el principio de que toda propiedad natural aumenta la semejanza entre los particulares que las ejemplifican.

Después de estas consideraciones resulta conveniente hacer una breve recapitulación general. En primer lugar, hemos presentado una análisis de las propiedades en términos de relaciones de semejanza entre particulares que otorgaba a ciertos particulares fijados, los paradigmas, un papel especial en la determinación de la propiedad, $\mathrm{y}$ también hemos visto dos de los principales problemas específicos de esta versión: la sobredeterminación de paradigmas y el problema de la independencia. A continuación, hemos considerado toda una serie de alternativas a la versión con paradigmas, que parten de la noción germinal de círculo de semejanza de Carnap. Estas versiones deben afrontar el problema de la coextensión, cuya 
solución debe pasar por un modalización de la teoría. También hemos visto cómo incluso muchas de las versiones modalizadas no consiguen evitar el problema de la comunidad imperfecta, a excepción de la propuesta de Hirsch, que, como se ha argumentado, tiene también sus propias dificultades, una de las cuáles es, ciertamente, de amplio alcance. Para acabar, comentaremos una objeción de Russell que atañe a todas las versiones del nominalismo de semejanza vistas hasta aquí, tanto a las que apelan a paradigmas, como a las que emplean la noción de círculo de semejanza, o alguna otra afín, aunque Russell formula objeción para una versión del análisis de semejanza con paradigmas ${ }^{22}$.

La objeción de Russell se puede exponer del modo siguiente. Consideremos cierta colección de esmeraldas, todas las cuales presentan exactamente el mismo tono de verde. En una u otra versión, el nominalismo de semejanza acaba explicando el color de estas esmeraldas en términos de relaciones de semejanza entre ellas. El hecho de que las esmeraldas presenten el mismo tono de verde hace que estas esmeraldas se asemejen entre sí en color exactamente de la misma manera. Ahora, o bien decimos que las relaciones presentes en los diferentes ejemplos pares de esmeraldas - son la misma relación, o bien decimos que se trata de relaciones diferentes. Sin embargo, si decimos que se trata de relaciones diferentes, todavía deberíamos admitir que se asemejan entre sí, pues no en vano los tonos de verde eran idénticos, de forma que las semejanzas consiguientes deberán tener algo en común. $Y$ ahora la situación se reproduce en relación a estas semejanzas postuladas entre las relaciones originales del primer nivel. $\mathrm{O}$ bien se trata de la misma relación que conecta todas los ejemplos de semejanza relacional, o bien se trata de relaciones diferentes pero semejantes, etc. A cada estadio, podemos evitar un nuevo paso hacia la regresión infinita abandonando el análisis de semejanza a través del postulado de una relación que conecta todos sus ejemplos. Si se desea evitar el postulado de esta relación, reduciéndola a semejanzas, entonces no hacemos más que posponer el problema un paso más allá ${ }^{23,24}$.

22 RUSSELL 1912.

23 ARMSTRONG (1989, pp. 108-110) intenta defender el análisis de semjanza de esta regresión tratando de mostrar que todos los términos de la regresión supervienen en la relación original. Sin embargo, no nos parece una solución en modo alguno satisfactoria, pues la relación de superveniencia no permite un tratamiento reductivo de los términos supervenientes.

24 Quisiera agradecer a las siguientes personas sus comentarios a trabajos previos que dieron origen a este artículo: Ramon Cirera, José Díez, Manuel García-Carpintero, David Pineda y Josep Lluís Prades. Este artículo forma parte del proyecto de investigación PB95-0760 financiado por la DGICYT. 


\section{Bibliografía}

Armstrong, D. M., Universals and Scientific Realism. Vol I. Nominalism and Realism, Cambridge University Press, 1978.

- Universals and Scientific Realism. Vol II. A Theory of Universals, Cambridge University Press, 1978.

- Universals, Westview Press, 1989.

- A World of States of Affairs, Cambridge University Press, 1997.

CARnaP, R., The Logical Structure of the World Routledge, 1967.

GOODMAN, N., The Structure of Appearence, Bobbs-Merrill, 1966.

Hirsch, E., Dividing Reality, Oxford University Press., 1993.

PRICE, H. H., Thinking and Experience, Hutchinson, 1953.

RUsSelL, B., The Problems of Philosophy, Home University Library, 1912. 